\title{
Downregulation of long non-coding RNA B-Raf proto- oncogene-activated non-coding RNA reverses cisplatin resistance in laryngeal squamous cell carcinoma
}

Weiwei Han, Lin Niu, Lin Wang, Jixiang Liu, Huanying Li

Department of Otolaryngology Head and Neck Surgery, Tianjin Union Medicine Centre, Tianjin, China

Submitted: 29 September 2018; Accepted: 14 October 2018 Online publication: 31 December 2019

Arch Med Sci 2021; 17 (5): 1164-1174

DOI: https://doi.org/10.5114/aoms.2019.91352

Copyright (c) 2019 Termedia \& Banach

\section{Abstract}

Introduction: This study was performed to explore the function of B-Raf proto-oncogene-activated non-coding RNA (BANCR) in laryngeal squamous cell carcinoma (LSCC) and cisplatin resistance.

Material and methods: The relative expression level of long non-coding RNA (InCRNA) BANCR was examined by qRT-PCR in tumor tissues and adjacent tissues, normal laryngeal cells (Het-1A) and laryngeal squamous carcinoma cells (TU686, TU177). Cisplatin-resistant laryngeal squamous carcinoma cell lines (TU686-DDP-R, TU177-DDP-R) were established. Next, we inhibited BANCR expression by transfecting siRNA-BANCR and enhanced BANCR expression by transfecting pcDNA3.1-BANCR into TU686, TU177, TU686-DDP-R and TU177-DDP-R cells. The CCK-8 assay and clone formation assay were performed to detect colony proliferation ability and formation ability of cells. Further, to investigate through which BANCR cell viability/formation is regulated, we detected the expression of MRP1, Bcl-2, p-PKB, and Bax by western blot.

Results: BANCR was highly expressed in laryngeal squamous carcinoma tissues and cells. Chemoresistance was generated in TU686-DDP-R and TU177-DDP-R compared with TU686 and TU177 cells after cisplatin treatment. In addition, upregulated IncRNA BANCR reduced or postponed cell sensitivity to cisplatin by enhancing cell proliferation in TU686 and TU177 cells. Meanwhile, the expression of MRP1, Bcl-2, and p-PKB was increased, while Bax was reduced. After cisplatin treatment, down-regulation of BANCR could consequently attenuate TU686-DDP-R and TU177-DDP-R cell proliferation, and the expression of MRP1, Bcl-2, and p-PKB was decreased and Bax was increased.

Conclusions: Down-regulation of BANCR reverses cisplatin resistance of cisplatin-resistant LSCC cell lines.

Key words: laryngeal squamous cell carcinoma, B-Raf proto-oncogeneactivated non-coding RNA, cisplatin, resistance.

\section{Introduction}

Laryngeal squamous cell carcinoma (LSCC) is the one of the most common cancers within men worldwide, increasing by about 600,000 cases per year [1], which is caused by drinking and smoking and other possible risk factors such as human papilloma virus (HPV) and Epstein-Barr virus (EBV) infections, work environment and nutrition [1, 2]. A survey

\author{
Corresponding author: \\ Weiwei Han \\ Department of Otolaryngology \\ Head and Neck Surgery \\ Tianjin Union Medicine Centre \\ 130 Jieyuan Road \\ Hongqiao District \\ Tianjin, 300110, China \\ Phone/fax: \\ +86-022-27561632 \\ E-mail: xuiqin163@163.com
}


shows that there are about 9,500-11,000 new laryngeal cancer patients in the United States [3] At present, larynx-conserving surgery and radiotherapy are effective strategies aiming at the therapy of the cancer, and combined therapy acts on advanced laryngeal cancer, including adjuvant therapy $[4,5]$. However, the mortality rate of LSCC remains high [6]. Under this circumstance, some researchers have studied the biomarkers of LSCC. Schluter et al. reported that CD31 and vascular endothelial growth factor (VEGF) are prognostic biomarkers [7]. Other studies indicated that miR-93-5p [8] and miR-196b [9] are potential markers in LSCC. However, studies on long non-coding RNAs (InCRNA) are rare in LSCC, while IncRNA is a critical regulator of coding RNA and alternative splicing, and it is associated with tumorigenesis, tumor progression, and metastasis [10]. Therefore, it is particularly urgent to search for new biomarkers of LSCC to carry out early diagnosis.

Long non-coding RNAs are RNA molecules that are between 200 and 100,000 nucleotides (nt) long and which are incapable of being translated into proteins $[11,12]$. Research has shown that IncRNAs regulated crucial cancer pathways at epigenetic, transcriptional, and post-transcriptional levels [13]. Meanwhile, reports showed that abnormal IncRNA expression has been found in tumors. The IncRNA HEIH showed higher transcript levels in hepatitis $B$ virus-related hepatocellular carcinoma (HCC). The IncRNA JADE has markedly higher levels in human breast tumors. Simultaneously, aberrant transcript profiles of IncRNAs were observed in non-neoplastic diseases, such as ventricular septal defect and acute renal rejection [14]. Numerous studies have indicated that IncRNA was involved in the regulation of various processes in the cell, such as cell growth, proliferation, differentiation, survival and apoptosis $[15,16]$. Therefore, scientists started to discover that imbalance of IncRNAs leads to the evolution of tumor through adjusting these cell processes [13, 17]. Besides, IncRNA expression had not only cellularity and tissue specificity, but also some IncRNA were expressed only in specific stages of eukaryotic development [18].

B-Raf proto-oncogene (BRAF)-activated non-coding RNA (BANCR) has been confirmed to have a close relationship with some human cancers [19]. Research indicated that BANCR has abnormal expression in some cancers, such as colorectal cancer [20], melanoma [21], gastric cancer [22], lung carcinoma [23], and hepatocellular carcinoma [24]. For example, Shi et al. found that BANCR inhibited colorectal cancer cell growth via interaction with p21 protein, and it demonstrated that BANCR was a regulator of the pathogenesis in colorectal cancer [20]. Also, BANCR promoted cell proliferation in lung carcinoma and malignant melanoma by regulating the mitogen-activated protein kinase (MAPK) pathway $[23,25]$. Above all, BANCR was a potential biomarker of early diagnosis, prognosis, and potential therapy targets in laryngeal carcinoma [26]. However, the expression and function of InCRNA BANCR in LSCC are unclear.

Cisplatin has been widely used in many solid tumors, is one of the most effective and common drugs for the treatment of a wide spectrum of tumors, and patients usually get effective results in the early stages of using cisplatin [27]. However, the overuse of therapy accelerated the process of generating drug tolerance, leading to an obstacle for a favorable therapeutic outcome [28]. After pathogens, such as HPV and EBV, make contact with the drug many times, the sensitivity of tumor cells was reduced or even disappeared, resulting in the reduction or invalidation of the drug to the pathogens [2, 29]. Drug resistance of tumor cells is the central barrier for the medical domain to treat laryngeal carcinoma, and continuous use of the drug often hinders treatment results $[16,30]$.

In this study, we altered BANCR expression by transfection to explore the role of BANCR in the progression of laryngeal carcinoma cell. The results revealed that downregulation of BANCR expression enhanced cell sensitivity to cisplatin via inhibiting cell proliferation of LSCC.

\section{Material and methods}

\section{Tissue samples and cell culture}

In total, 47 specimens of human LSCC tissues and adjacent tissues were obtained from the Tianjin Union Medicine Centre (Tianjin, China). Samples were allocated into two groups: an experimental group (tumor tissues) and a control group (adjacent tissues). The study was performed according to the institutional ethical guidelines. The ethics committee of Tianjin Union Medicine Centre approved a written informed consent form which was signed by every patient, and all the consents were saved by the ethics committee. The conduct of the study was in accordance with the principles expressed in the Declaration of Helsinki. All samples were snap-frozen in liquid nitrogen, then stored at $-80^{\circ} \mathrm{C}$ for further use.

Normal esophageal cell Het-1A and laryngeal squamous carcinoma cells TU686 and TU177 came from BeNa Culture Collection (Beijing, China). Cells were cultivated in Roswell Park Memorial Institute (RPMI) 1640 culture medium supplemented with $10 \%$ fetal bovine serum (FBS, TBD, Tianjin, China), 100 units $/ \mathrm{ml}$ penicillin $\mathrm{G}$ and $100 \mu \mathrm{g} / \mathrm{ml}$ streptomycin and incubated in a $5 \% \mathrm{CO}_{2}$ humidified atmosphere at $37^{\circ} \mathrm{C}$. Cisplatin (DDP)-resistance (R) laryngeal squamous carcinoma cells (TU686-DDP-R, 
TU177-DDP-R) came from TU686 and TU177 cells with continuous cisplatin treatment.

\section{Western blotting}

Cells were harvested and lysed in radioimmunoprecipitation assay buffer. Protein samples were separated by sodium dodecyl sulfate polyacrylamide gel electrophoresis and transferred onto polyvinylidene difluoride (PVDF) membranes. After blocking with $5 \%$ skimmed milk, the membranes were incubated overnight at $4^{\circ} \mathrm{C}$ with primary antibodies, followed by incubation with secondary antibodies.

\section{Quantitative reverse transcription-} polymerase chain reaction (QRT-PCR) assay

Total RNA was isolated from tissues and cells using TRIzol reagent (Invitrogen, Carlsbad, CA, USA). All steps were performed under RNasefree condition. A UV spectrophotometer (BioRad, California, USA) was used to detect RNA concentration and optical density (OD) value. The reverse transcription reactions were carried out via a PrimeScript RT reagent kit (RR047A, TaKaRa Bio Inc., Japan). For quantitative real-time PCR (qPCR), a final volume of $20 \mu \mathrm{l}$ reaction was performed according to a standard protocol and the SYBR Green PCR kit (Roche Diagnostics, Indianapolis, IN, USA) on the StepOnePlus Real-Time PCR System (Applied Biosystems, Carlsbad, CA, USA). The GPCR was performed in triplicate, with no template controls. The $2^{-\Delta \Delta C T}$ method was conducted to determine the relative gene expression levels and the endogenous control was glyceraldehyde-3-phosphate dehydrogenase (GAPDH). The QRT-PCR was performed using the following cycles: $95^{\circ} \mathrm{C}$ for $30 \mathrm{~s} ; 40$ cycles of $95^{\circ} \mathrm{C}$ for $5 \mathrm{~s}$ and $60^{\circ} \mathrm{C}$ for $31 \mathrm{~s}$; and a dissociation stage at $95^{\circ} \mathrm{C}$ for $15 \mathrm{~s}, 60^{\circ} \mathrm{C}$ for $1 \mathrm{~min}$ and $95^{\circ} \mathrm{C}$ for $15 \mathrm{~s}$. The following were the primer (Sangon biotech, Shanghai) sequences: BANCR primers: 5'-ACAGGACTCCATGGCAAACG-3' (forward) and 5'-ATGAAGAAAGCCTGGTGCAGT-3' (reverse). GAPDH primers: 5'-GCACCGTCAAGGCTGAGAAC-3' (forward) and 5'-TGGTGAAGACGCCAGTGGA-3' (reverse).

\section{SiRNA and plasmid transfection}

The specific small interfering RNA that targeted BANCR (siRNA-BANCR) and a scrambled negative control (siRNA-NC) came from GenePharma (Shanghai, China). The expression vector (pcDNA3.1) expressing BANCR (pcDNA3.1-BANCR) and a scrambled negative control (pcDNA3.1) were also constructed and purchased from GenePharma (Shanghai, China). The cells were cultured $24 \mathrm{~h}$ prior to transfection. Then, the cells were transiently transfected with corresponding siRNA or pcDNA3.1 by using Lipofectamine 2000 Transfection Reagent (Invitrogen, Carlsbad, CA, USA) on the basis of the manufacturer's instructions. Cells transfected with siRNA or pcDNA3.1-BANCR were harvested for further analysis after 48 h. Small interfering RNAs can guide Argonaute-containing RNA-induced silencing complexes to target RNAs in a sequence-specific way, leading to mRNA deadenylation followed by exonucleolytic decay, mRNA endonucleolytic cleavage, or translational inhibition [31]. pcDNA 3.1 is $5.4 \mathrm{~kb}$ vector and is designed for high-level expression. For high-level expression, it contains the human cytomegalovirus (CMV) immediate-early promoter. High-level expression can be performed in most mammalian cells [32].

\section{Cell counting}

The cells were cultured $24 \mathrm{~h}$ prior to transfection. Cells were treated with expressing BANCR (pcDNA3.1-BANCR), a scrambled negative control (pcDNA3.1) and cultured for $48 \mathrm{~h}$, then cells were collected through trypsinization, mixed in PBS and trypan blue was added. Cells were counted using a microscope counting chamber.

\section{CCK-8 assay}

A reagent of Cell Counting Kit-8 (CCK-8) assay (Beyotime, Institute of Biotechnology, Shanghai, China) was used in the cell proliferation assay. 3000 viable cells per well a 96-well tissue culture plates in a final volume of $100 \mu \mathrm{l}$ were used. Every $24 \mathrm{~h}$, a plate was subjected to assay by adding $10 \mu \mathrm{l}$ of CCK-8 solution to each well, and the plate was further incubated for $4 \mathrm{~h}$ at $37^{\circ} \mathrm{C}$. The Microplate Photometer was used to measure the absorbance at $450 \mathrm{~nm}$. All assays were performed in triplicate and all experiments were repeated three times.

\section{Colony formation assay}

The method of the colony formation assay: a total of 1000 cells were plated into each well of a 6 -well plate and cultured in medium containing $10 \% \mathrm{FBS}$ incubation at $37^{\circ} \mathrm{C}$ in $5 \% \mathrm{CO}_{2}$ for 2 weeks, replacing the medium every 4 days. The colonies were fixed with methanol, stained with Giemsa, and counted. Triplicate wells were measured in each treatment group.

\section{Statistical analysis}

Data were analyzed using GraphPad Prism 6 and were expressed as the mean $\pm S D$. The significance of the differences between groups was estimated by Student's t-test, or one-way analysis of variance, as appropriate. Two-sided $p<0.05$ was deemed to show a statistically significant difference. 


\section{Results}

\section{LncRNA BANCR expression level was high in tumor tissues and LSCC cells}

The relative BANCR expression level was detected in tumor tissues and adjacent normal tissues from 47 patients via using QRT-PCR. It was notable that BANCR expression was higher in tumor tissues compared with adjacent normal tissues (Figure 1 A). Compared with the Het-A cells, the BANCR expression was increased significantly in LSCC cells TU686 and TU177 (Figure 1 B). To evaluate LSCC cell growth after overexpression BANCR, we estimated cell counts. As shown in Figure $1 \mathrm{C}$, the cell count was significantly higher in the pcDNA3.1-BANCR group, compared with the pcDNA3.1 group. In order to confirm the prognostic value of BANCR expression for laryngeal squamous cell carcinoma, we acquired the Kaplan-Meier survival curves for the correlation between the levels of BANCR expression and overall survival from The Cancer Genome Atlas (TCGA), and we found that BANCR expression was signifi- cantly correlated with laryngeal squamous cell carcinoma patients' overall survival (Figure 1 D). Patients with increased BANCR expression had worse overall survival than those with low expression of BANCR. The above data demonstrated that IncRNA BANCR may function as an actuator gene in LSCC. Thus, it can be seen that BANCR had a certain relationship with LSCC.

\section{The level of BANCR was overexpressed} in cisplatin-resistant LSCC cells

Initially, we established cisplatin-resistant cell lines TU686-DDP-R and TU177-DDP-R. TU686 and TU686-DDP-R cells, and TU177 and TU177DDP-R cells were treated with various concentrations of $\operatorname{DDP}(1,2,4,8,16,32 \mu \mathrm{g} / \mathrm{ml})$ for $48 \mathrm{~h}$. Then, the cell viability was examined (Figures $2 \mathrm{~A}$, B). The results showed that cell viability of TU686 was significantly lower compared with TU686DDP-R cells with DDP treatment. Similar results appeared in TU177 cells and TU177-DDP-R cells. Colony formation assays indicated that the colo-
A

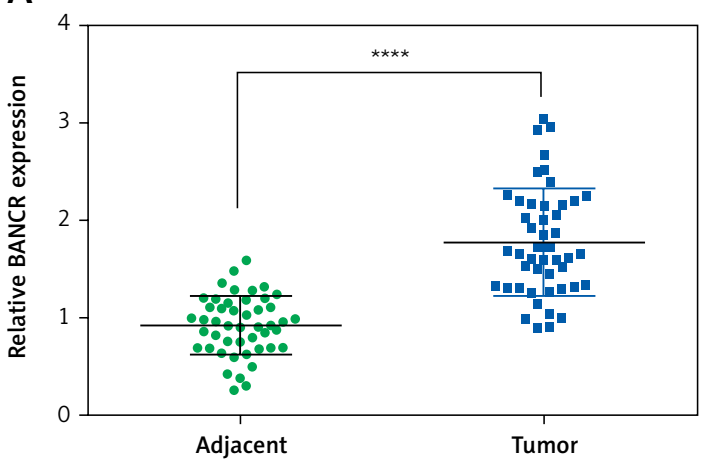

C

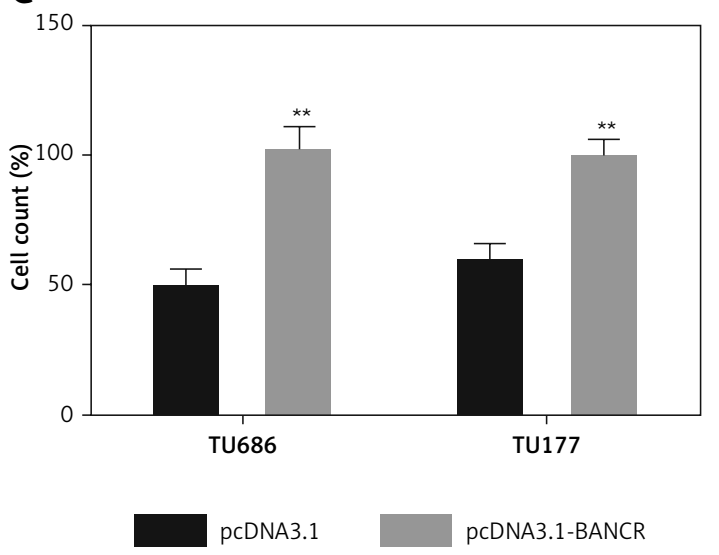

B

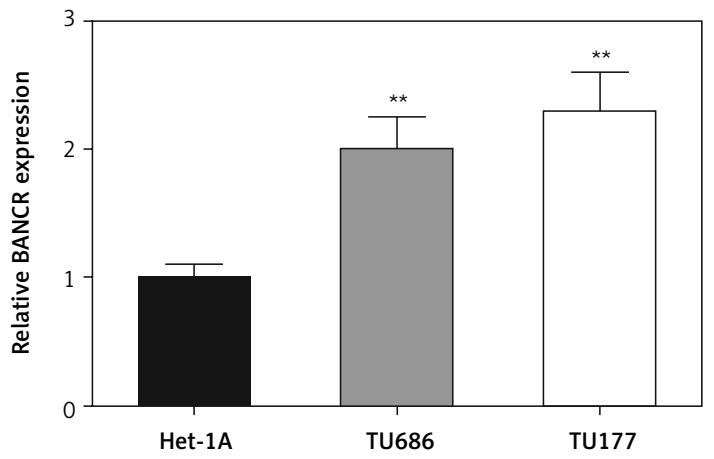

D

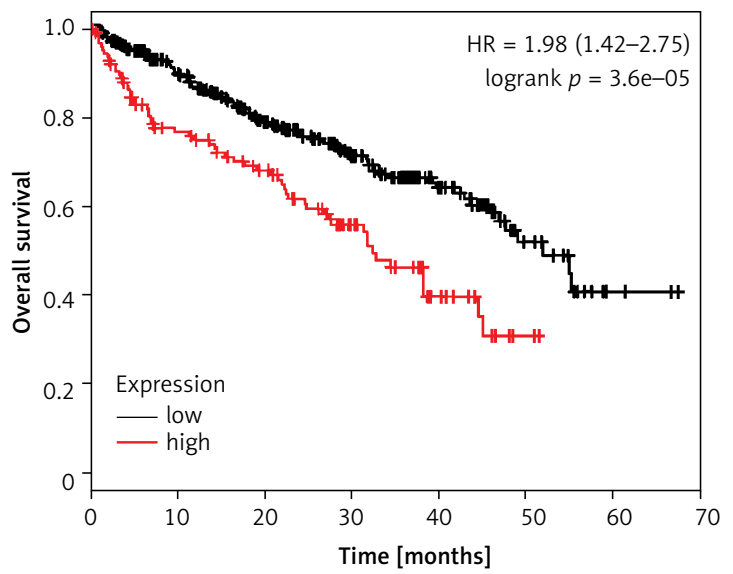

Figure 1. Expression of BANCR in tumor tissues and laryngeal squamous cell carcinoma (LSCC) cells. A - The expression of BANCR was examined by qRT-PCR, and 47 samples of tumor tissues and adjacent tissues were investigated. ${ }^{* \star *} P<0.0001$, compared with adjacent tissues. B - Expression of BANCR in LSCC cells and laryngeal cells was determined by qRT-PCR. ${ }^{* *} P<0.01$, compared with Het-1A. C - Cell counting after pcDNA3.1-BANCR transfection in TU686 and TU177 cell lines. Cell count was markedly increased in pcDNA3.1-BANCR group, compared with pcDNA3.1 control. D - The association between overall survival rates and BANCR expression 
A

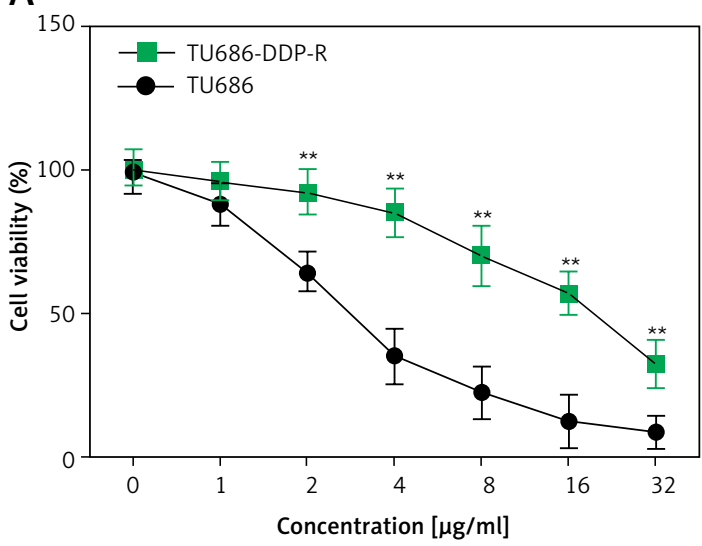

B

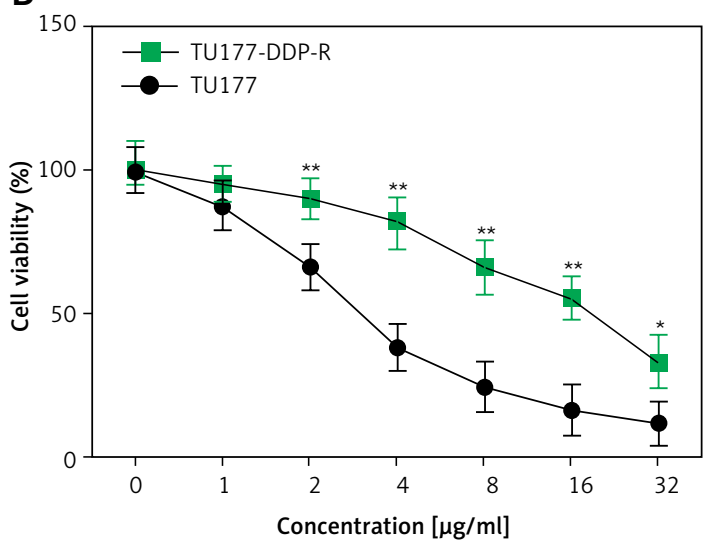

C

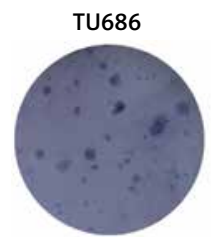

TU686-DDP-R

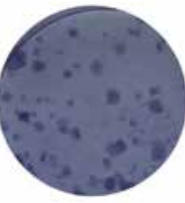

TU177

TU177-DDP-R
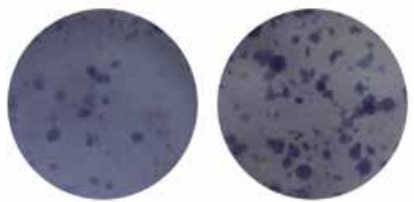

D

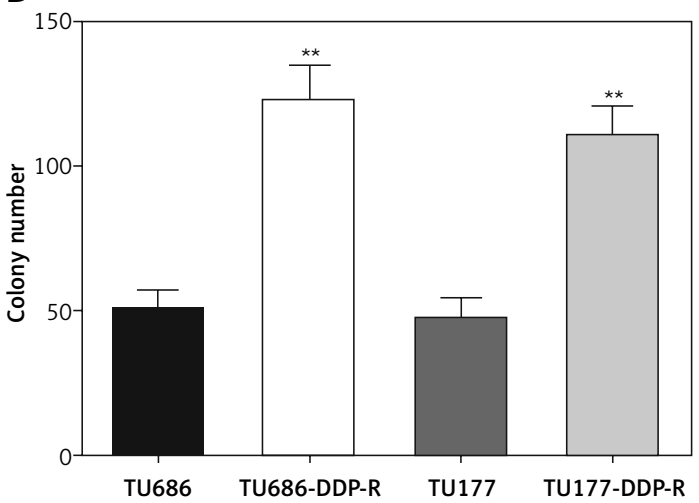

$\mathrm{E}$

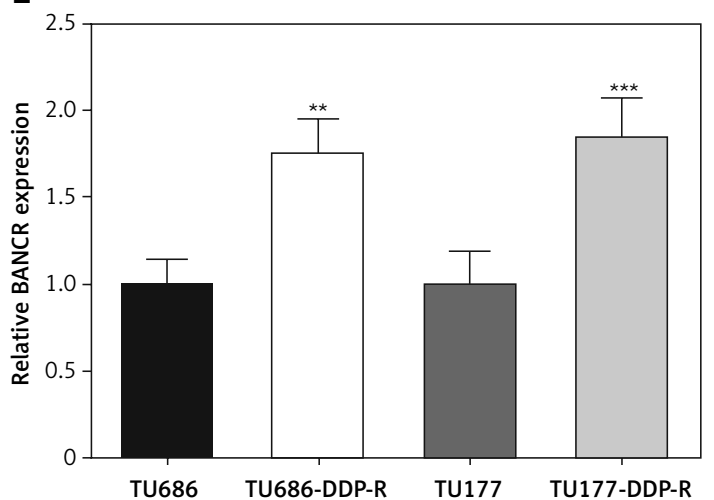

Figure 2. The cisplatin-resistant cell line was established. A, B - TU686 and TU686-DDP-R cells, and TU177 and TU177-DDP-R cells were treated with various concentrations of DDP $(0,1,2,4,8,16,32 \mu \mathrm{g} / \mathrm{ml})$. C, D - Colonyforming growth assays were performed to determine the proliferation of TU686, TU177, TU686-DDP-R, TU177DDP-R cells with $10 \mu \mathrm{g} / \mathrm{ml}$ DDP treatment. The colonies were counted and captured. $\mathbf{E}-$ The expression of BANCR in TU686, TU686-DDP-R, TU177, TU177-DDP-R was observed by qRT-PCR assay. Data represent the mean \pm SD. ${ }^{* *} P<0.01$, compared with TU686 or TU177

ny numbers of TU686-DDP-R and TU177-DDP-R cells with $10 \mu \mathrm{g} / \mathrm{ml}$ DDP treatment were obviously higher than TU686 and TU177 (Figures 2 C, D). As shown in Figure $2 \mathrm{E}$, we observed that the expression of BANCR in TU686-DDP-R and TU177-DDP-R was significant higher compared with TU686 and TU177. It indicated that high BANCR expression may make cells become resistant to cisplatin in LSCC.

\section{Downregulation of BANCR increased} sensitivity of cisplatin-resistant LSCC cells by inhibiting proliferation in vitro

BANCR expression levels were enhanced by transfecting pCDNA-BANCR in TU686 and TU177 cells, while the expression of BANCR was inhibited via transfecting with siRNA-BANCR in TU686- 
A

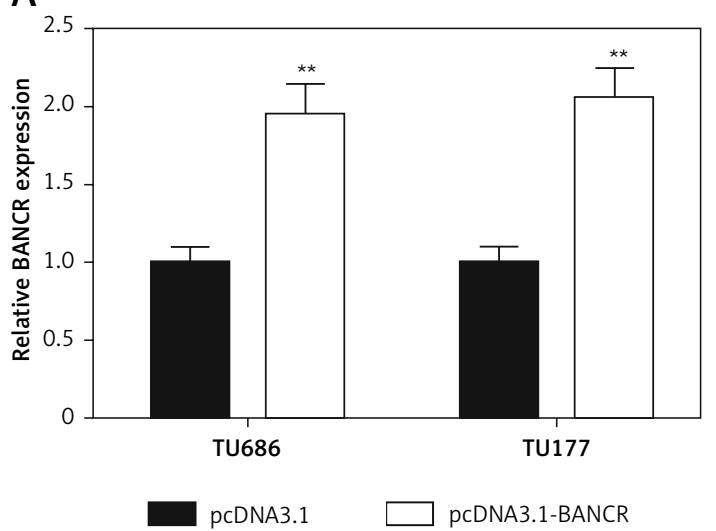

C
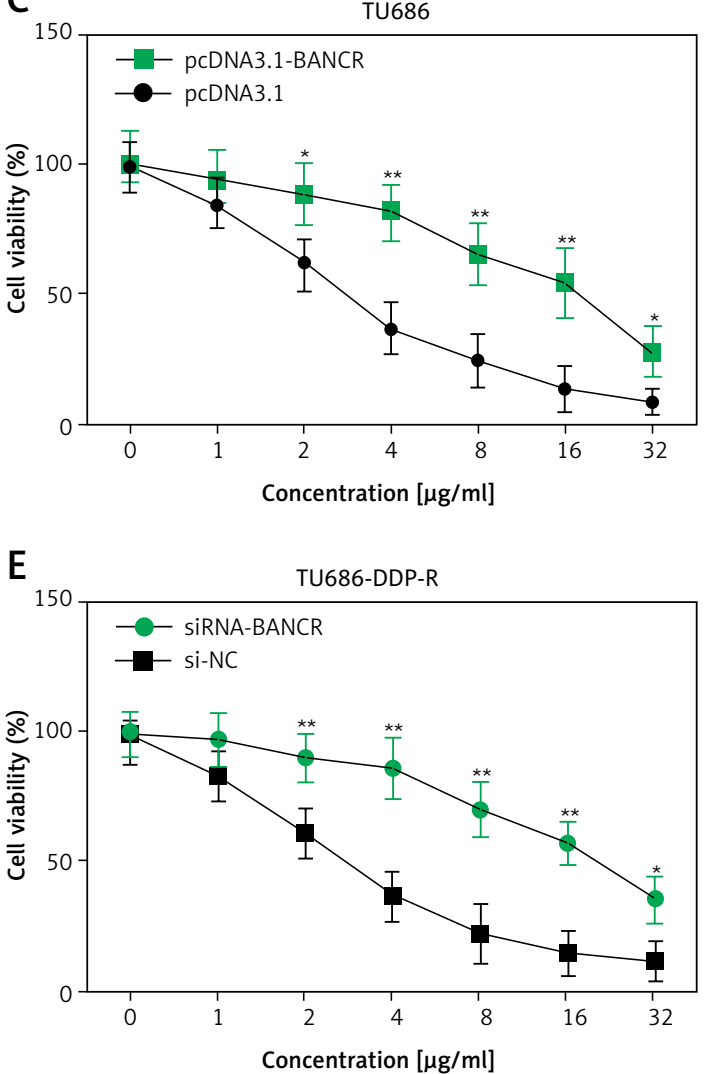

B

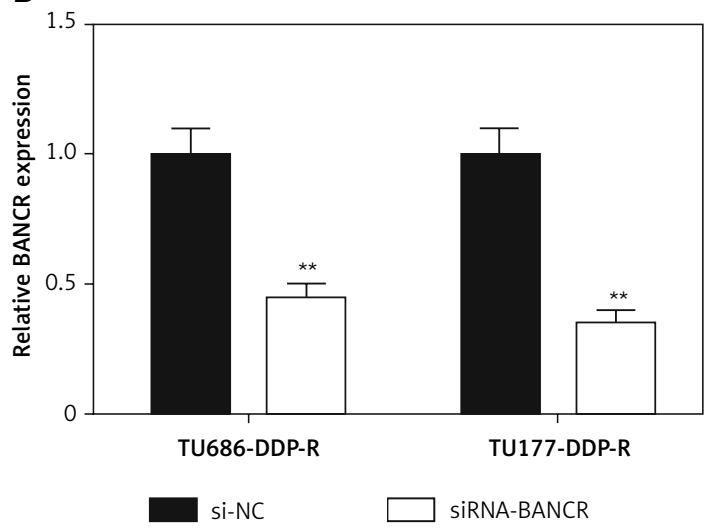

D

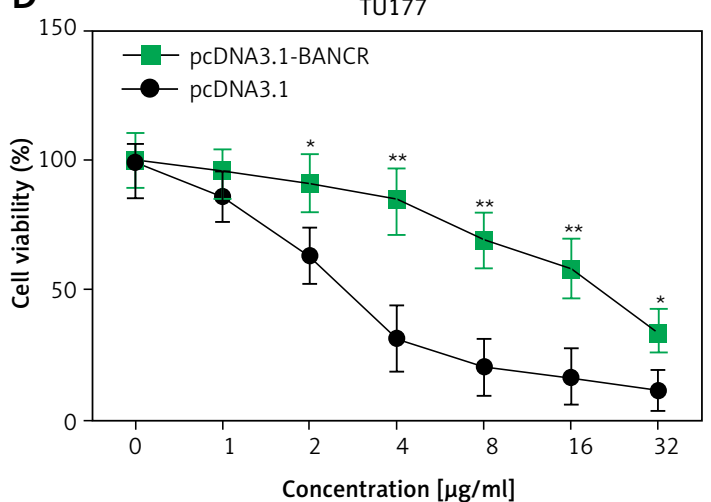

$\mathrm{F}$

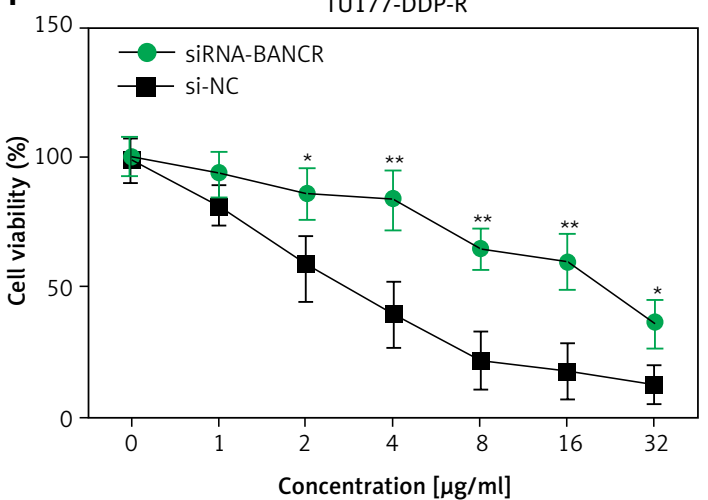

Figure 3. The effect of BANCR expression on sensitive cells and cisplatin-resistant cells in laryngeal squamous cell carcinoma (LSCC). A - Relative expression of BANCR in TU686 and TU177 after transfection of pCDNA3.1 and pcDNA3.1-BANCR. ${ }^{* *} P<0.01$, compared with pcDNA3.1. B - Relative BANCR expression of TU686-DDP-R, TU177DDP-R after transfection of siRNA-BANCR and si-NC. ${ }^{* *} P<0.01$, compared with si-NC. C, D - CCK-8 assay was used to evaluate sensitivity of TU686 and TU177 to DDP after transfection of pcDNA3.1-BANCR. All cells were administered with different concentrations of cisplatin for $24 \mathrm{~h} .{ }^{* *} P<0.01$, compared with pcDNA3.1. E, F - CCK-8 assay was used to evaluate sensitivity of TU686-DDP-R and TU177- DDP-R to DDP after transfection SiRNA-BANCR. ${ }^{* *} P<0.01$, compared with si-NC

DDP-R and TU177-DDP-R. QRT-PCR analysis of BANCR expression levels suggested that BANCR expression was enhanced in TU686 and TU177 cells following transfection with pcDNA-BANCR compared with pcDNA3.1 (Figure 3 A). Relative expression levels of BANCR in TU686-DDP-R and TU177-DDP-R with siRNA-BANCR were fewer than
si-NC (Figure $3 \mathrm{~B}$ ). Moreover, cell viability of LSCC cells with pcDNA3.1-BANCR transfection was significantly high following treatment with various concentrations of $\operatorname{DDP}(1,2,4,8,16,32 \mu \mathrm{g} / \mathrm{ml})$ compared with the pcDNA3.1 group (Figures $3 \mathrm{C}, \mathrm{D}$ ), while cell viability of DDP-resistant LSCC cells with siRNA-BANCR was clearly lower compared with 
A

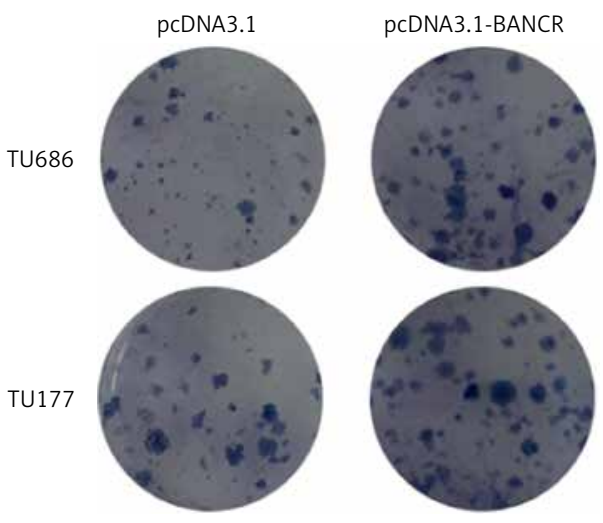

B

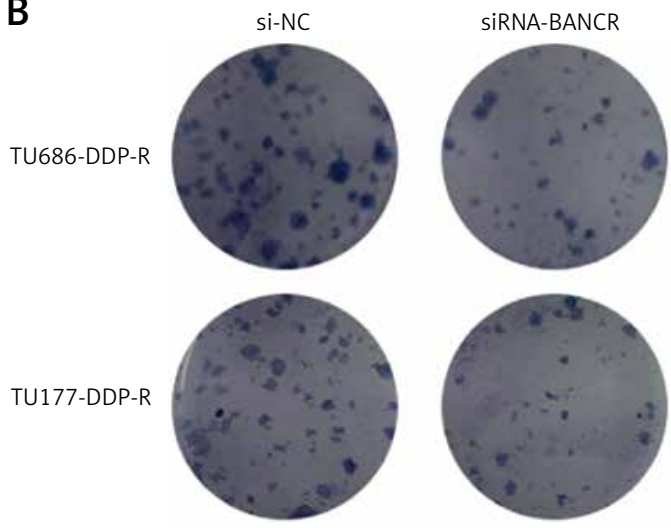

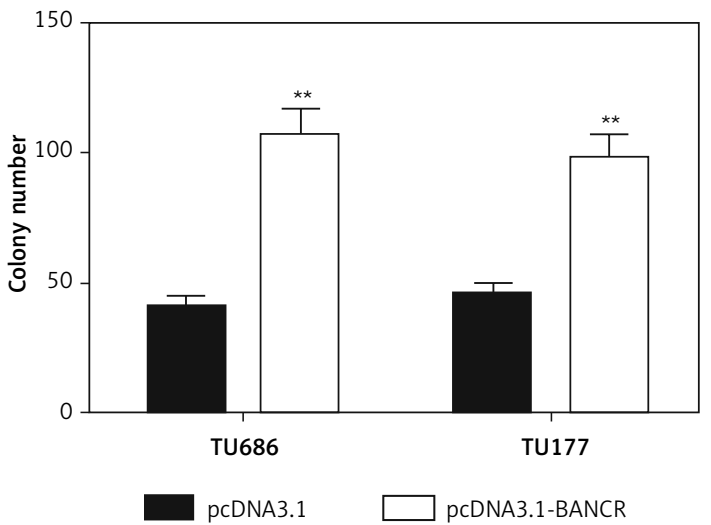

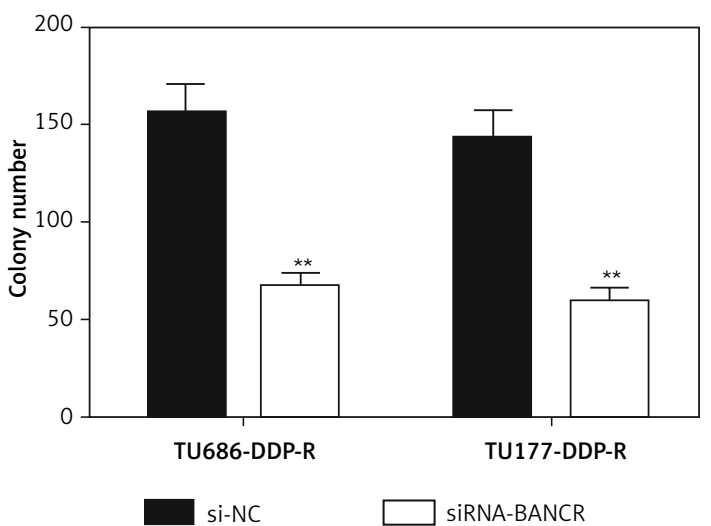

Figure 4. Sensitivity of cells to DDP was examined by colony-forming growth assays. A - The level of clone formation in TU686 and TU177 with pcDNA3.1-BANCR or pCDNA3.1 transfection and DDP treatment in vitro. The colonies were counted and captured. ${ }^{* *} P<0.01$, compared with pcDNA3.1. B - After treatment with siRNA-BANCR and DDP, the colonies of TU686-DDP-R and TU177-DDP-R were counted. ${ }^{* *} P<0.01$, compared with si-NC. All cells were treated with $10 \mu \mathrm{g} / \mathrm{ml}$ DDP

si-NC (Figures 3 E, F). Representative data of experiments showed that upregulated BANCR reduced sensitivity of TU686 and TU177 cell to cisplatin. In contrast, the cisplatin-resistant cell line became sensitive after downregulation of BANCR expression.

\section{Downregulation of BANCR increased sensitivity of cisplatin-resistant LSCC cells by inhibiting colony formation in vitro}

The proliferative impact of BANCR on the process of cells was further assessed by colony formation assays. Colony numbers of the pcDNA3.1BANCR group were obviously higher than the pcDNA3.1 in TU686 and TU177 cells with $5 \mu \mathrm{g} / \mathrm{ml}$ DDP treatments. However, colony numbers of the siRNA-BANCR group were lower than si-NC in TU686-DDP-R and TU177-DDP-R with $5 \mu \mathrm{g} / \mathrm{ml}$ DDP treatment (Figures 4 A, B). The observations indicated that downregulation of BANCR increased sensitivity of cisplatin-resistant LSCC cells.

\section{Modulation of MRP1 and apoptosis-related genes}

To investigate through which BANCR cell viability/formation is regulated, we detected the expression of MRP1, Bcl-2, p-PKB and Bax. Bcl-2 is anti-apoptotic protein, while Bax plays an important role in proapoptotic processes. They exist in intrinsic apoptotic pathways [33]. Further, protein kinase $B(P K B)$ is an important member of the PI3/PKB pathway. The activated PI3K/PKB pathway was found in head and neck carcinomas and phosphorylated protein kinase B (p-PKB) was increased [34]. Moreover, multidrug resistance is a major contributor to the failure of chemotherapy in patients suffering from multidrug resistance-associated protein 1 (MRP1) was up-regulated in laryngeal cancer $[35,36]$. Our results showed that the expression of MRP1, Bcl-2, p-PKB of LSCC cells with pcDNA3.1-BANCR transfection was significantly high compared with the pcDNA3.1 group (Figures $5 \mathrm{~A}-\mathrm{C}, \mathrm{E}-\mathrm{G}$ ), while the expression of $\mathrm{Bax}$ 

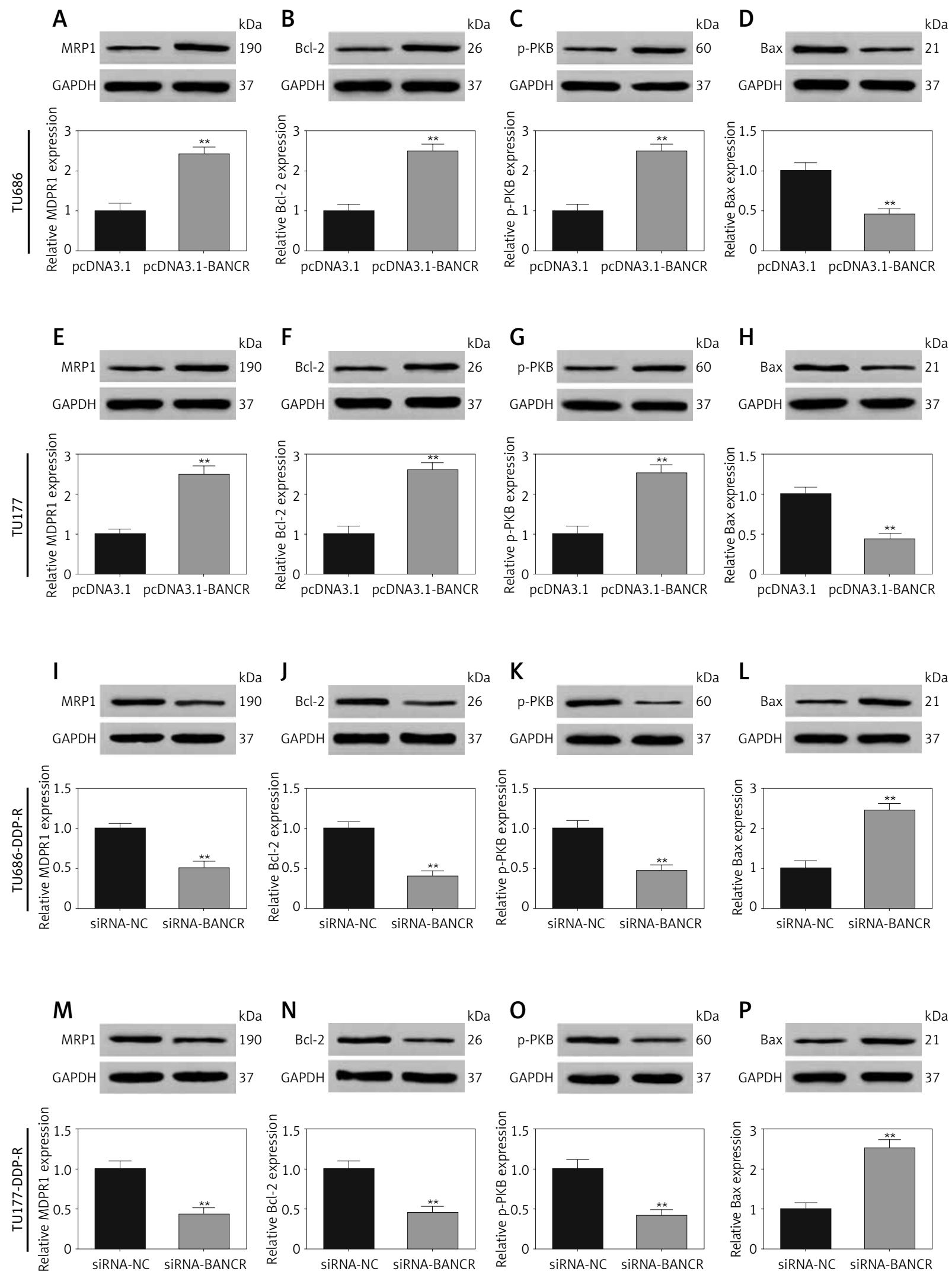

Figure 5. The effect of BANCR on the expression of MRP1, Bcl-2, p-PKB, Bax in sensitive cells and cisplatin-resistant cells of laryngeal squamous cell carcinoma (LSCC). The expression of MRP1, Bcl-2, p-PKB, Bax in TU686 (A-D) and TU177 (E-H) with pcDNA3.1-BANCR or pcDNA3.1 transfection. " $P<0.01$, compared with pcDNA3.1. MRP1, Bcl-2, p-PKB and Bax expression in TU686-DDP-R (I-L) and TU177-DDP-R (M-P) after transfection of siRNA-BANCR or siNC. ${ }^{* *} P<0.01$, compared with si-NC 
was decreased in the pcDNA3.1-BANCR group (Figures $5 \mathrm{D}, \mathrm{H}$ ). In addition, MRP1, Bcl-2, and $\mathrm{p}$-PKB expression was reduced in DDP-resistant LSCC cells with siRNA-BANCR compared with si-NC (Figures $5 \mathrm{I}-\mathrm{K}, \mathrm{L}-\mathrm{N}$ ). Conversely, the Bax expression was increased (Figures $5 \mathrm{E}, \mathrm{O}$ ). These results indicated that the PI3/PKB signaling pathway may be related to cell viability, which was regulated by BANCR. $\mathrm{BCl}-2$ and Bax may participate in this process.

\section{Discussion}

Laryngeal squamous carcinoma is the most common cancer of head and neck squamous cell carcinoma around the world [6, 37], in which IncRNAs occupy an important position. Feng et al. reported that IncRNAs are potential biomarkers in advanced LSCC [38]. Wu et al. showed that LnCRNA H19 could accelerate LSCC pathological processes through miR-148a-3p and DNM [39]. Li et al. indicated that IncRNA HOTAIR plays a regulatory role in LSCC [40]. At present, relevant research has found that IncRNA may have a vital effect on different aspects of cell pathology and physiology, such as cancer [41]. Cisplatin (DDP) has a wide anticancer spectrum, strong function, and it has no cross resistance or other characteristics, such as toxicity, and cisplatin can establish a mouse model of acute kidney injury [42]. Further, it is one of the most commonly used drugs in combination chemotherapy at present. However, drug resistance is a serious obstacle to efficient chemotherapy and represents a major problem in clinical oncology [27]. Consequently, gene therapy becomes an appealing and effective option for cancer treatment. The experiments of Zhu et al. demonstrated that the suppression of the cluster of differentiation 147 (CD147) gene as a potential target for therapeutic anti-cancer drugs enhanced chemosensitivity to cisplatin in laryngeal carcinoma human epithelial-2 (Hep2) cells [6]. Also, low LnCRNA HOTAIR expression promoted enhancer of zeste homolog 2 (EZH2) expression, contributing to the increase of the sensitivity to DDP in the LSCC cells through promoting the proliferation of AMC-HN8 cells [43]. Xu et al. deemed that apigenin may enhance the sensitivity to cisplatin of laryngeal carcinoma cells via suppressing GLUT-1 and p-Akt genes [44]. Furthermore, some studies indicated IncRNAs, especially BANCR, as potential oncogenes or inhibitor genes to tumors, had crucial regulatory functions in tumorigenesis and tumor progression. Zhang et al. reported that BANCR as a tumor suppressor inhibits aggressiveness in papillary thyroid cancer [45]. Moreover, Wang et al. indicated that BANCR promotes endometrial cancer cell proliferation, migration and invasion [46]. In addition, BANCR had an inseparable relationship with melanomas, papillary thyroid carcinomas, colorectal carcinomas, lung cancers and serous ovarian carcinomas [47, 48], which means that BANCR may serve as a novel biomarker for cancer diagnosis and disease prognosis evaluation. BANCR-targeting inventions may be explored as a promising therapeutic option in cancer treatments [49]. That is, regulating BANCR was a possible orientation to treat tumor, and IncRNA BANCR can be considered as therapeutic targets, at least for some cancer types [50]. However, it was completely unclear for the biological functions of IncRNA BANCR in LSCC.

Research revealed that high BANCR expression emerged in LSCC cells. Similarly, BANCR was overexpressed in malignant melanoma tissues [21]. Conversely, low expression of BANCR had been observed in both lung cancers and colorectal cancers [23]. Also, the research of He et al. revealed that BANCR expression was low in bladder cancer tissues and cells [51]. We have developed a cisplatin-resistant LSCC cell line from human laryngeal carcinoma cells TU686 and TU177 with cisplatin treatment, showing that BANCR was overexpressed in cisplatin-resistant cell lines TU686-DDP-R and TU177-DDP-R based on BANCR overexpressed in LSCC cells. Further, we regulated expression of BANCR to explore whether BANCR expression had an impact on sensitivity of LSCC cells and cisplatin-resistant LSCC cells by influencing cell proliferation after the transfection of pcDNA3.1-BANCR or siRNA-BANCR. Previous research showed that downregulation of BANCR promoted the proceeding of proliferation via inhibiting p21 protein expression in colorectal cancer cells and bladder cancer cells $[20,51]$. On the other hand, BANCR accelerated cell proliferation via activating the MAPK pathway in lung carcinoma $[25,52]$. Furthermore, down-regulation of BANCR remarkably repressed endometrial cancer cell proliferation via inhibiting the ERK/MAPK signaling pathway that regulates MMP1 and MMP2 [45]. In our research, apparently, on the one hand, elevated BANCR expression protected LSCC cells from the toxicity of cisplatin and promoted cell proliferation in comparison with the control cells. On the other hand, the results showed that knockdown of BANCR repressed proliferation of LSCC cells. Meanwhile, overexpression BANCR increased the expression of p-PKB in LSCC cells, while knockdown of BANCR decreased protein expression levels of $p$-PKB in resistant cells. Taken together, the results showed that downregulation of BANCR enhanced sensitivity of cisplatin-resistant LSCC cells by inhibiting proliferation in vitro. The PI3/PKB signaling pathway may participate in this process.

In conclusion, BANCR was confirmed to participate in the progression of cells such as proliferation in laryngeal squamous carcinoma. Knockdown 
of BANCR restrained cell proliferation. Our study demonstrated that sensitivity of cisplatin-resistant laryngeal squamous carcinoma cells could be rescued successfully by downregulating the expression of IncRNA BANCR. The study of specific BANCR biological function has important significance for the diagnosis of cancer and prediction of new target genes, and our studies provide an important theoretical basis for further research.

\section{Conflict of interest}

The authors declare no conflict of interest.

\section{References}

1. Solomon B, Young RJ, Rischin D. Head and neck squamous cell carcinoma: genomics and emerging biomarkers for immunomodulatory cancer treatments. Semin Cancer Biol 2018; 52: 228-40.

2. Guan GF, Zhang DJ, Wen LJ, et al. Overexpression of IncRNA H19/miR-675 promotes tumorigenesis in head and neck squamous cell carcinoma. Int J Med Sci 2016; 13: 914-22.

3. Ampil FL, Nathan CO, Caldito G, Lian TF, Aarstad RF, Krishnamsetty RM. Total laryngectomy and postoperative radiotherapy for T4 laryngeal cancer: a 14-year review. Am J Otolaryngol 2004; 25: 88-93.

4. Johung K, Rewari A, Wu H, et al. Role of excision repair cross-complementation 1 expression as a prognostic marker for response to radiotherapy in early-stage laryngeal cancer. Head Neck 2013; 35: 852-7.

5. Liu T, Peng H, Zhang M, Deng Y, Wu Z. Cucurbitacin B, a small molecule inhibitor of the Stat3 signaling pathway, enhances the chemosensitivity of laryngeal squamous cell carcinoma cells to cisplatin. Eur J Pharmacol 2010; 641: 15-22.

6. Zhu C, Pan Y, He B, et al. Inhibition of CD147 gene expression via RNA interference reduces tumor cell invasion, tumorigenicity and increases chemosensitivity to cisplatin in laryngeal carcinoma Hep2 cells. Oncol Rep 2011; 25: 425-32.

7. Schluter A, Weller P, Kanaan O, et al. CD31 and VEGF are prognostic biomarkers in early-stage, but not in latestage, laryngeal squamous cell carcinoma. BMC Cancer 2018; 18: 272.

8. Shan $\mathrm{X}$, Zhang $\mathrm{H}$, Zhang L, et al. Identification of four plasma microRNAs as potential biomarkers in the diagnosis of male lung squamous cell carcinoma patients in China. Cancer Med 2018; 7: 2370-81.

9. Zhao X, Zhang W, Ji W. miR-196b is a prognostic factor of human laryngeal squamous cell carcinoma and promotes tumor progression by targeting SOCS2. Biochem Biophys Res Commun 2018; 501: 584-92.

10. Wang X, Yu H, Sun W, et al. The long non-coding RNA CYTOR drives colorectal cancer progression by interacting with NCL and Sam68. Mol Cancer 2018; 17: 110.

11. Ponting CP, Oliver PL, Reik W. Evolution and functions of long noncoding RNAs. Cell 2009; 136: 629-41.

12. Mercer TR, Dinger ME, Mattick JS. Long non-coding RNAs: insights into functions. Nat Rev Genet 2009; 10: 155-9.

13. Geng YJ, Xie SL, Li Q, Ma J, Wang GY. Large intervening non-coding RNA HOTAIR is associated with hepatocellular carcinoma progression. J Int Med Res 2011; 39: 2119-28.
14. Wu Z, Liu X, Liu L, et al. Regulation of IncRNA expression. Cell Mol Biol Lett 2014; 19: 561-75.

15. Martens-Uzunova ES, Bottcher R, Croce CM, Jenster G, Visakorpi T, Calin GA. Long noncoding RNA in prostate, bladder, and kidney cancer. Eur Urol 2014; 65: 1140-51.

16. Tsai MC, Spitale RC, Chang HY. Long intergenic noncoding RNAs: new links in cancer progression. Cancer Res 2011; 71: 3-7.

17. Nie Y, Liu X, Qu S, Song E, Zou H, Gong C. Long non-coding RNA HOTAIR is an independent prognostic marker for nasopharyngeal carcinoma progression and survival. Cancer Sci 2013; 104: 458-64.

18. Zou Y, Li J, Chen Y, et al. BANCR: a novel oncogenic long non-coding RNA in human cancers. Oncotarget 2017; 8: 94997-5004.

19. Flockhart RJ, Webster DE, Qu K, et al. BRAFV600E remodels the melanocyte transcriptome and induces BANCR to regulate melanoma cell migration. Genome Res 2012; 22: 1006-14.

20. Shi Y, Liu Y, Wang J, et al. Downregulated long noncoding RNA BANCR promotes the proliferation of colorectal cancer cells via downregualtion of $\mathrm{p} 21$ expression. PLoS One 2015; 10: e0122679.

21. Li R, Zhang L, Jia L, et al. Long non-coding RNA BANCR promotes proliferation in malignant melanoma by regulating MAPK pathway activation. PLoS One 2014; 9: e100893.

22. Zhang ZX, Liu ZQ, Jiang B, et al. BRAF activated non-coding RNA (BANCR) promoting gastric cancer cells proliferation via regulation of NF-kappaB1. Biochem Biophys Res Commun 2015; 465: 225-31.

23. Jiang $W$, Zhang $D, X u B$, et al. Long non-coding RNA BANCR promotes proliferation and migration of lung carcinoma via MAPK pathways. Biomed Pharmacother 2015; 69: 90-5

24. Zhou T, Gao Y. Increased expression of LnCRNA BANCR and its prognostic significance in human hepatocellular carcinoma. World J Surg Oncol 2016; 14: 8.

25. Staff PO. Correction: Long non-coding RNA BANCR promotes proliferation in malignant melanoma by regulating MAPK pathway activation. PLoS One 2015; 10: e0118728.

26. Chandra Gupta S, Nandan Tripathi Y. Potential of long non-coding RNAs in cancer patients: from biomarkers to therapeutic targets. Int J Cancer 2017; 140: 1955-67.

27. Cimbora-Zovko T, Fritz G, Mikac N, Osmak M. Downregulation of RhoB GTPase confers resistance to cisplatin in human laryngeal carcinoma cells. Cancer Lett 2010; 295: 182-90.

28. Kelland L. The resurgence of platinum-based cancer chemotherapy. Nat Rev Cancer 2007; 7: 573-84.

29. Chen W, Hu Q, Liu WE. The microbiology and clinical characteristics of bacterial and fungal meningitis in a Chinese hospital. Arch Med Sci Civil Dis 2016; 1: e99-e109.

30. Liu HN, Qie P, Yang G, Song YB. miR-181b inhibits chemoresistance in cisplatin-resistant $\mathrm{H} 446$ small cell lung cancer cells by targeting Bcl-2. Arch Med Sci 2018; 14: 745-51.

31. Kim YJ, Maizel A, Chen X. Traffic into silence: endomembranes and post-transcriptional RNA silencing. EMBO J 2014; 33: 968-80.

32. Guo F, Tang C, Li Y, et al. The interplay of LncRNA ANRIL and miR-181b on the inflammation-relevant coronary artery disease through mediating NF-kappaB signalling pathway. J Cell Mol Med 2018; 22: 5062-75.

33. Wu C, Luo H, Ma W, et al. Polysaccharides isolated from Hedyotis diffusa inhibits the aggressive phenotypes 
of laryngeal squamous carcinoma cells via inhibition of Bcl-2, MMP-2, and muPA. Gene 2017; 637: 124-9.

34. Brolih S, Parks SK, Vial V, et al. AKT1 restricts the invasive capacity of head and neck carcinoma cells harboring a constitutively active PI3 kinase activity. BMC Cancer 2018; 18: 249.

35. Jehle J, Muller CFH, Aksoy A, Zimmer S, Nickenig G, Tiyerili $V$. Genetic disruption of multidrug resistance-associated protein 1 improves endothelial function and attenuates atherosclerosis in MRP1(-/-) LDLr(-/-) double knockout mice. Arch Med Sci 2017; 13: 930-6.

36. Li D, Zhou L, Huang J, Xiao X. Effect of multidrug resistance 1/P-glycoprotein on the hypoxia-induced multidrug resistance of human laryngeal cancer cells. Oncol Lett 2016; 12: 1569-74.

37. Klisic A, Kavaric N, Jovanovic M, Soldatovic I, Gligorovic-Barhanovic N, Kotur-Stevuljevic J. Bioavailable testosterone is independently associated with Fatty Liver Index in postmenopausal women. Arch Med Sci 2017; 13: 1188-96.

38. Feng L, Wang R, Lian M, et al. Integrated analysis of long noncoding RNA and mRNA expression profile in advanced laryngeal squamous cell carcinoma. PLoS One 2016; 11: e0169232.

39. Wu T, Qu L, He G, et al. Regulation of laryngeal squamous cell cancer progression by the IncRNA H19/miR148a-3p/DNMT1 axis. Oncotarget 2016; 7: 11553-66.

40. Li D, Feng J, Wu T, et al. Long intergenic noncoding RNA HOTAIR is overexpressed and regulates PTEN methylation in laryngeal squamous cell carcinoma. Am J Pathol 2013; 182: 64-70

41. Qiu MT, Hu JW, Yin R, Xu L. Long noncoding RNA: an emerging paradigm of cancer research. Tumour Biol 2013; 34: 613-20.

42. Li J, Tang Y, Tang PMK, et al. Blocking macrophage migration inhibitory factor protects against cisplatin-induced acute kidney injury in mice. Mol Ther 2018; 26: 2523-32.

43. Zheng J, Xiao X, Wu C, et al. The role of long non-coding RNA HOTAIR in the progression and development of laryngeal squamous cell carcinoma interacting with $\mathrm{EZH} 2$. Acta Otolaryngol 2017; 137: 90-8.

44. Xu YY, Wu TT, Zhou SH, et al. Apigenin suppresses GLUT-1 and $\mathrm{p}$-AKT expression to enhance the chemosensitivity to cisplatin of laryngeal carcinoma Hep-2 cells: an in vitro study. Int I Clin Exp Pathol 2014; 7: 3938-47.

45. Zhang J, Du Y, Zhang X, Li M, Li X. Downregulation of BANCR promotes aggressiveness in papillary thyroid cancer via the MAPK and PI3K pathways. J Cancer 2018; 9: 1318-28.

46. Wang D, Wang D, Wang N, Long Z, Ren X. Long non-coding RNA BANCR promotes endometrial cancer cell proliferation and invasion by regulating MMP2 and MMP1 via ERK/MAPK signaling pathway. Cell Physiol Biochem 2016; 40: 644-56.

47. Roberts PJ, Der CJ. Targeting the Raf-MEK-ERK mitogenactivated protein kinase cascade for the treatment of cancer. Oncogene 2007; 26: 3291-310.

48. Rajagopalan H, Bardelli A, Lengauer C, Kinzler KW, Vogelstein B, Velculescu VE. Tumorigenesis: RAF/RAS oncogenes and mismatch-repair status. Nature 2002; 418: 934.

49. Liu XF, Hao JL, Xie T, et al. The BRAF activated non-coding RNA: a pivotal long non-coding RNA in human malignancies. Cell Prolif 2018; 51: e12449.

50. Yu X, Zheng H, Chan MT, Wu WKK. BANCR: a cancer-related long non-coding RNA. Am J Cancer Res 2017; 7: 1779-87.

51. He A, Liu Y, Chen Z, et al. Over-expression of long noncoding RNA BANCR inhibits malignant phenotypes of human bladder cancer. J Exp Clin Cancer Res 2016; 35: 125.
52. Sun M, Liu XH, Wang KM, et al. Downregulation of BRAF activated non-coding RNA is associated with poor prognosis for non-small cell lung cancer and promotes metastasis by affecting epithelial-mesenchymal transition. Mol Cancer 2014; 13: 68 